\title{
La gestion des risques au bloc opératoire. Le facteur humain et la culture sécurité.
}

\section{Dr Romero G}

Centre Léon Bérard - Lyon

L'erreur médicale est encore un tabou dans les pays Anglo-Saxons comme en France, alors que le nombre D'EIG (événement indésirable grave) est significatif. De plus, 30\% des complications seraient dues à une erreur médicale (Fabri et al. Surgery 2008), entraînant près de 100000 décès par an aux USA (Wong et al. JBJS AM 2009) avec un impact financier de 29 milliards de Dollars (Robinson et al. JBJS Br 2009). En France, d'après l'étude ENEIS 2 (2009) près de $100000 \mathrm{EIG}$ surviendraient dans la période peri opératoire dont près de la moitié serait évitable.

C'est dans ce contexte qu'il faut comprendre les notions de danger et de risques, afin de les traiter et les maitriser au mieux dans le cadre de la prise en charge des patients. Ainsi, le danger est un facteur qui peut induire un évènement indésirable (El) pouvant entrainer un préjudice pour un patient ; et le risque correspond à la probabilité de survenue de cet évènement ; ce qui peut être mesuré en gravité et en probabilité de survenue. Sur cela s'ajoute une notion d'activité humaine et de prise de risque dans l'activité de soins, qui peut être volontaire ou involontaire, souvent en lien avec la performance. II existe plusieurs types d'El potentiellement source de dommage, qui peuvent aller du simple dysfonctionnement, un presqu'incident qui touche le patient sans conséquence, jusqu'à un accident. Quel que soit le type d'El, $80 \%$ d'entre eux ont une origine humaine, et lorsqu'un incident grave survient, il a été précédé dans $99 \%$ des cas, d'évènements précurseurs ; ainsi d'après la pyramide de Bird (Insurance Company of North America, 1969) il y a 30 incidents qui précèdent une catastrophe. La notion de risque et de danger ne suffit pas à expliquer la survenue d'un accident, qui correspond en fait à une combinaison d'événements aboutissant à l'accident et qui associe une situation dangereuse survenant au sein d'un système complexe correspondant à une structure de soins comme le bloc opératoire.

Se pose alors la question : « comment gérer les risques liés aux soins et pourquoi ? » . L'objectif majeur de la gestion des risques est d'assurer la sécurité des patients. Ainsi les risques en établissement doivent être traités selon 2 axes concernant les El à éviter (gestion des risques a priori), avec une cartographie des risques par exemple, et les El à gérer (gestion des risques a posteriori) selon une méthode d'analyse validée comme les RMM. L'analyse d'un El doit permettre de comprendre les causes et conséquences d'un accident. C'est à cette étape, que la notion d'erreur humaine doit être totalement intégrée et ainsi comprendre que les El se développent dans un contexte de causes profondes favorisant leur survenue (Berwick DM. Président et PDG, The Institute for Healthcare Improvement, dans une déclaration au Committee on Senate Appropriations Subcommittee of Labor, Health and Human Services, le 13 mars 2003). L'étape suivante doit permettre de hiérarchiser les risques selon leur niveau d'inacceptabilité, puis les traiter, c'est-à-dire rendre un risque acceptable.

Le traitement d'un risque lié aux soins a 4 mécanismes principaux, qui comprennent la suppression du risque, la prévention, la récupération et enfin l'atténuation en cas d'accident avéré. II existe également des outils clés en main afin de traiter au mieux les risques dans notre activité de soins comme la Check list HAS de sécurité au bloc (Haynes A. N Engl J Med 2009), Le CRM santé (Médical Team training, Wolf et al, Ann Surg 2010, Haller et al, Int J Qual Health Care 2008, Neily et al, JAMA 2010).

II existe d'autres clés pour la sécurité des soins entraînant des pratiques de fiabilité, qui demande cependant de détecter dans notre quotidien de soignant les situations à risque élevé d'erreur.

Au total, depuis près de 20ans la prise de conscience du facteur humain dans la genèse des El liés aux soins a permis de développer la culture sécurité des soins regroupant 10 dimensions majeures qui s'étendent du top management jusqu' au travail en équipe, en passant par la réponse non punitive de l'erreur, et notamment un apport plus récent dont le but est de devenir une équipe hautement fiable.

georges.romero@lyon.unicancer.fr

(C) The authors, published by EDP Sciences. This is an Open Access article distributed under the terms of the Creative Commons Attribution License 4.0 (http://creativecommons.org/licenses/by/4.0/). 